\title{
LHCb Front-End Electronics and Data Acquisition System
}

\author{
Beat Jost \\ Cern / EP \\ Presentation given at the \\ 7th International Conference on \\ Instrumentation for Colliding Beam Experiments \\ November 15-19, 1999 Hamamatsu, Japan
}




\section{IHCb Outline}

$\square$ Introduction

$\square$ Front-End Electronics

$>$ Functionality

$>$ Architecture

$\square$ Timing and Fast Control

$\checkmark$ Data Acquisition

$>$ Architecture

- Functional Components

$\square$ Controls

$\square$ Selected Topic

$>$ Event-Building Network Simulation

$\square$ Summary 


\section{KHCp Introduction to $\mathrm{LHCb}$}

- Special purpose experiment to measure precisely $C P$ violation parameters in the BB system and rare $B$ decays

- Detector is a single-arm spectrometer

- Total b-quark production rate is $\sim 75 \mathrm{kHz}$

- Expected rate from inelastic $p-p$ collisions is $\sim 15 \mathrm{MHz}$

- Branching ratios of interesting channels range between $10^{-5}-10^{-4}$ giving interesting physics rate of $\sim 5 \mathrm{~Hz}$

\section{$\mathrm{LHCb}$ in Numbers}

Number of Channels 950000 Bunch crossing rate $40 \mathrm{MHz}$ Level-0 accept rate $1 \mathrm{MHz}$ Level-1 accept rate $40 \mathrm{kHz}$ Readout Rate $40 \mathrm{kHz}$

Event Size $100 \mathrm{kB}$ Event Building Bandwidth $\quad 4 \mathrm{~GB} / \mathrm{s}$ Level-2 accept rate $\sim 5 \mathrm{kHz}$ Level-3 accept rate $\sim 200 \mathrm{~Hz}$ Level-2/3 CPU Power Data rate to Storage 


\section{IHCb LHCb Detector}

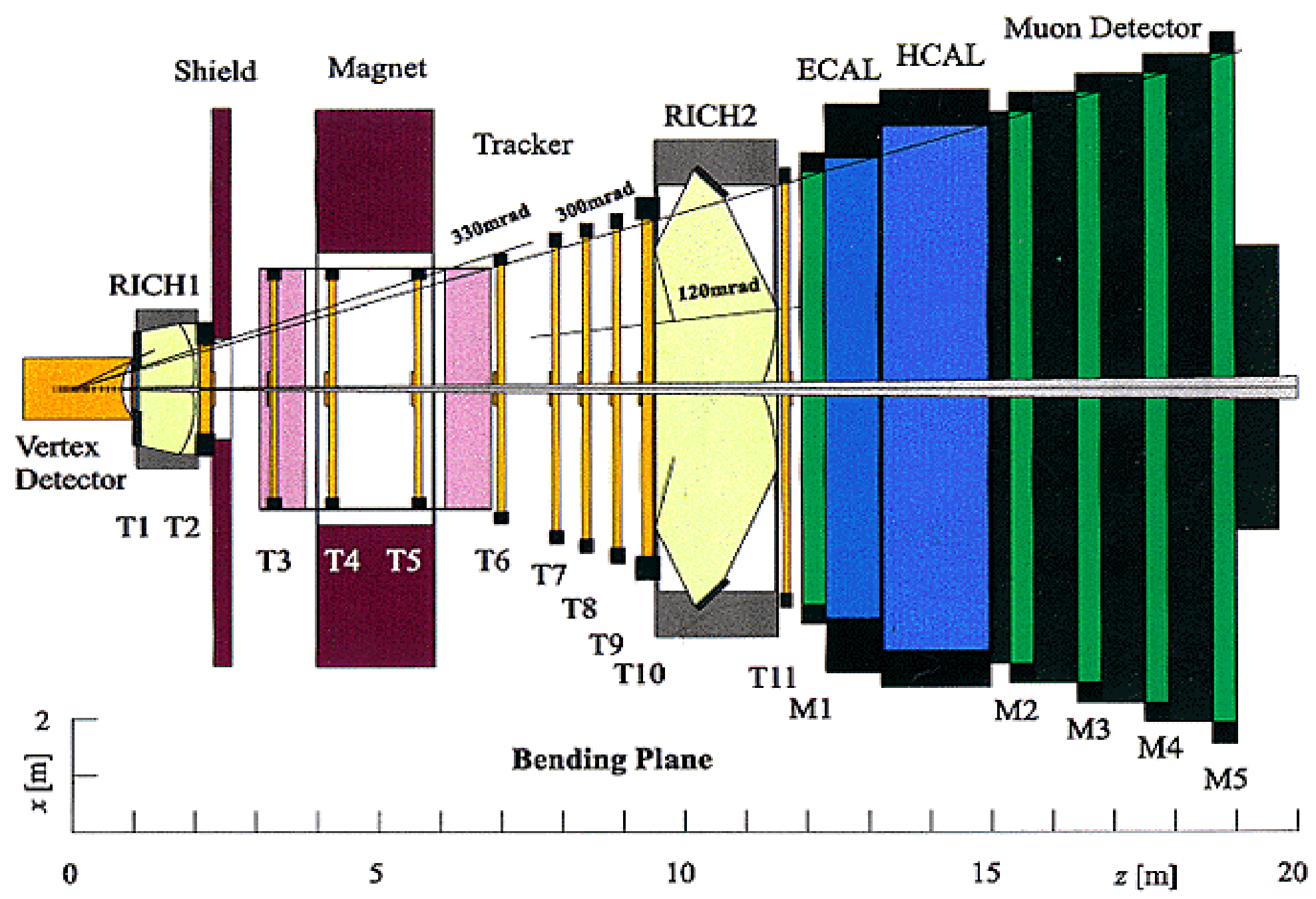




\section{KHCh Typical Interesting Event}

\section{triggered}

primary vertex
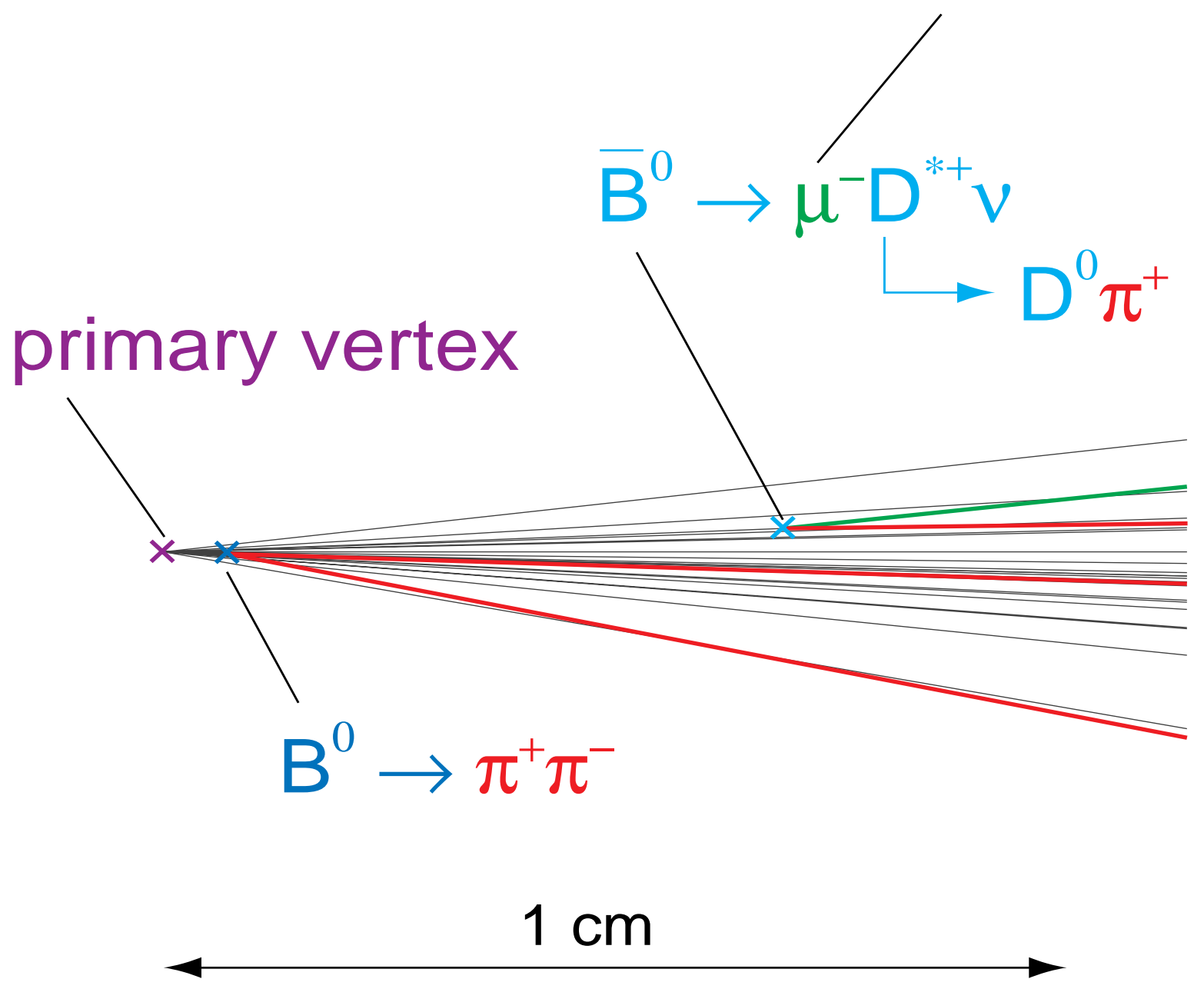


\section{LHCh Trigger/DAQ Architecture}

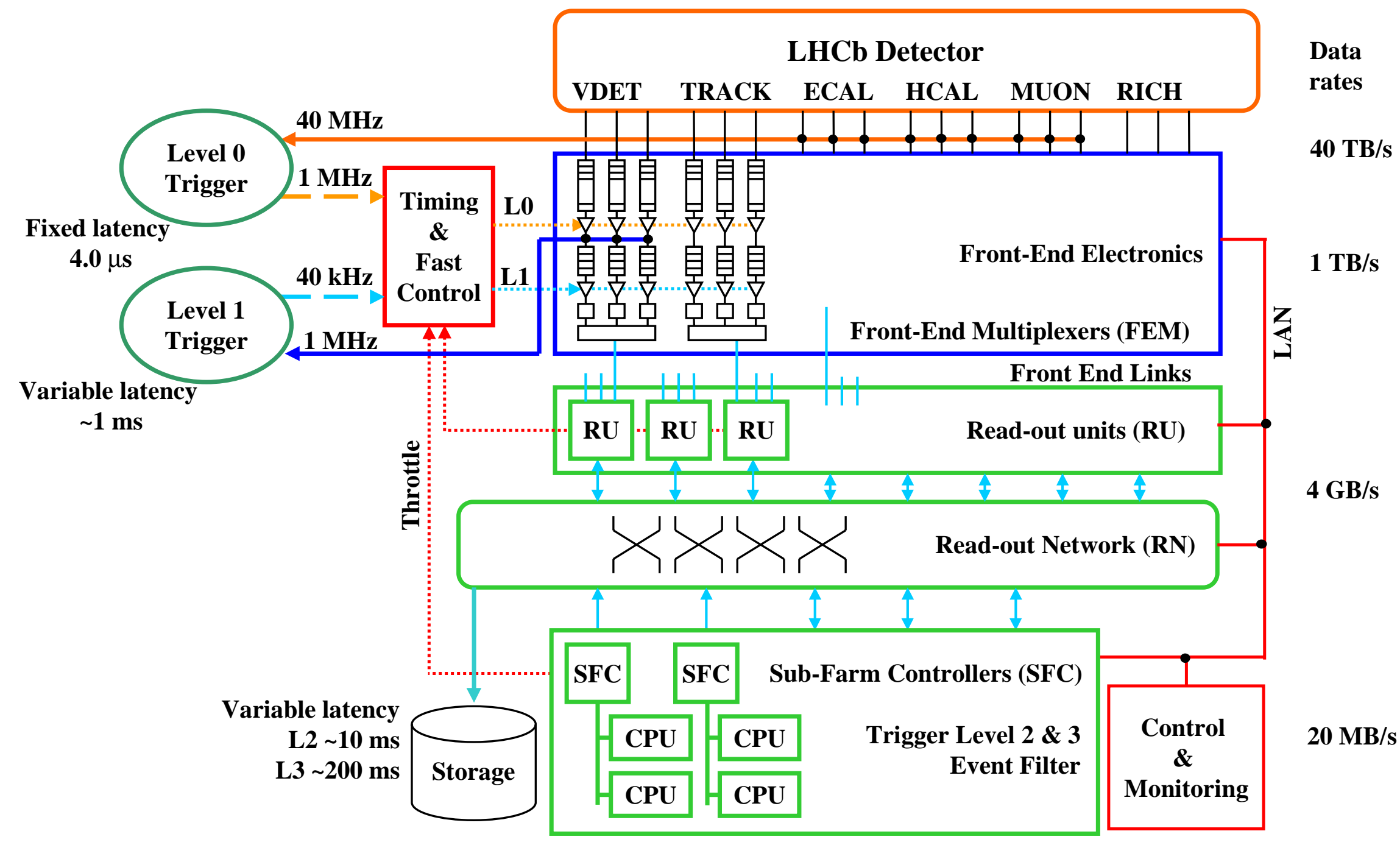




\section{KHCP Front-End Electronics}

LHCb has two levels of 'hardware' triggers

$\rightarrow$ Front-End Electronics has to buffer data twice for the corresponding latency

$\rightarrow$ Split Front-End Electronics into two parts

$\rightarrow$ Level-0 Electronics on or very close to the detector

$\rightarrow$ Level-1 Electronics near ( few meters away) the detector 


\section{LHCb Front-End Electronics Architecture and Functionality}

- Amplify/Shape detector signal

- Strobe detector data at Beam-Crossing Rate $(40 \mathrm{MHz})$

- Data buffering for Level-0 latency

- Digitization

- Data buffering for Level-1 latency

Zero suppression and data formatting

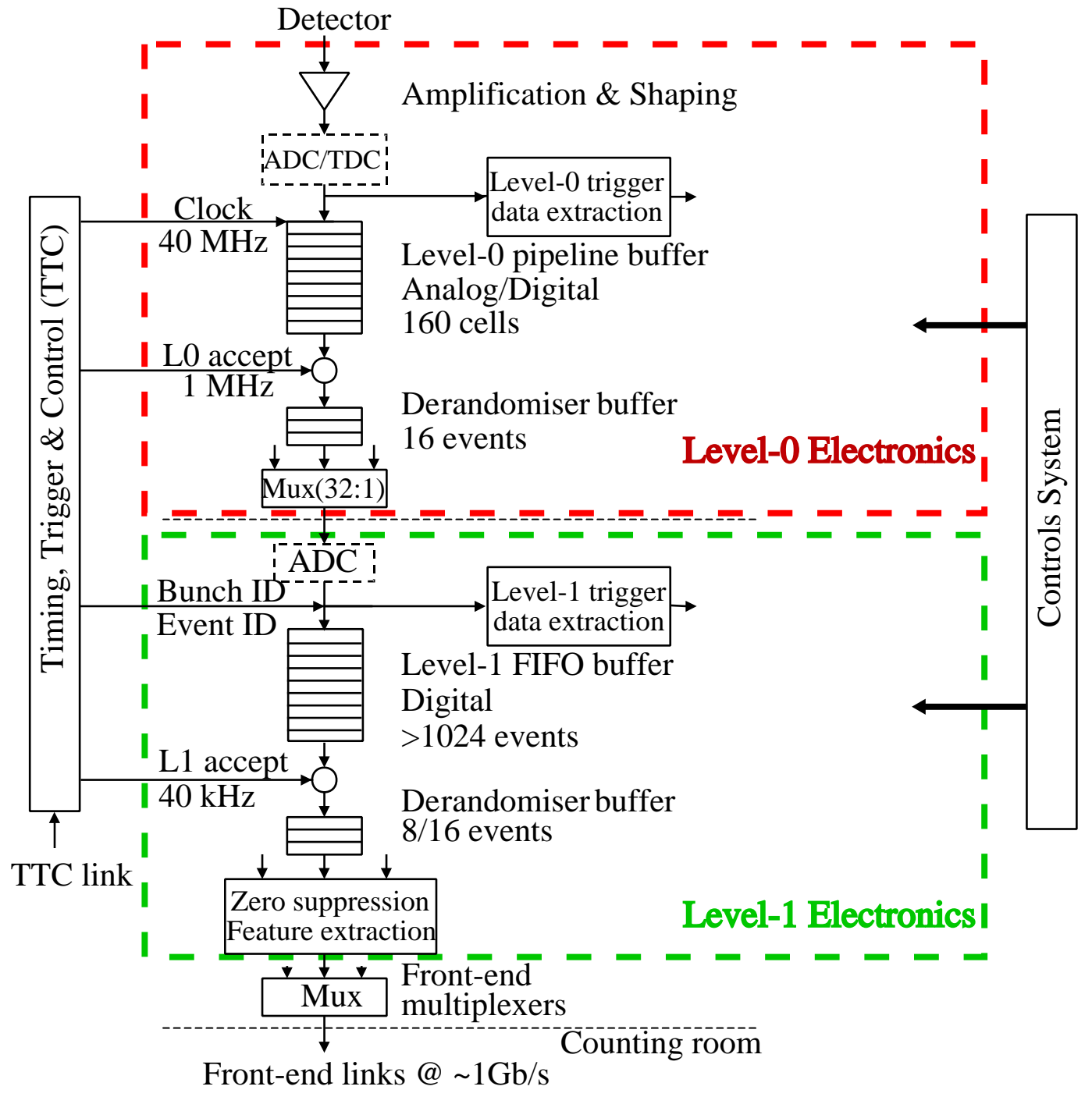




\section{KHCb LO Electronics Implementations}

\begin{tabular}{|c|c|c|c|}
\hline Detector & Signal & Candiate Chip(s) & Technology \\
\hline VELO & Analog (8bit) & Modified SCTA/Beetle & DMILL/0.25 $\mu$ \\
\hline Inner Tracker & Analog (8bit) & Modified SCTA/Beetle & DMILL/0.25 $\mu$ \\
\hline Outer Tracker & Time (digital, $0.5 \mathrm{~ns}$ ) & HPTDC & $0.25 \mu$ \\
\hline Rich $1 \& 2$ & $\begin{array}{c}\text { Analog (8bit)/ } \\
\text { Binary }\end{array}$ & $\begin{array}{l}\text { Modified SCTA/Beetle } \\
\text { Pixel Readout }\end{array}$ & DMILL/0.25 $\mu$ \\
\hline Preshower & Digital (8-10bit) & \multirow{4}{*}{ Hybrid } & \\
\hline ECal & Digital (12 bit) & & \\
\hline $\mathrm{HCal}$ & Digital (10 bit) & & \\
\hline Muon & Binary & & \\
\hline
\end{tabular}

Radiation Environments:

$>$ Velo: $1 \mathrm{Mrad} / \mathrm{y}$

> Others: <100 kRad/y

$>$ L1 Electronics: < 1kRad/y 


\section{LHCh Timing and Fast Control}

- Provide common and synchronous clock to all components needing it

- Provide Level-0 and Level-1 trigger decisions

- Provide commands synchronous in all components (Resets)

$\square$ Provide Trigger hold-off capabilities to prevent buffers from overflowing

- React to 'Throttles' from the readout system

$\checkmark$ Support Partitioning

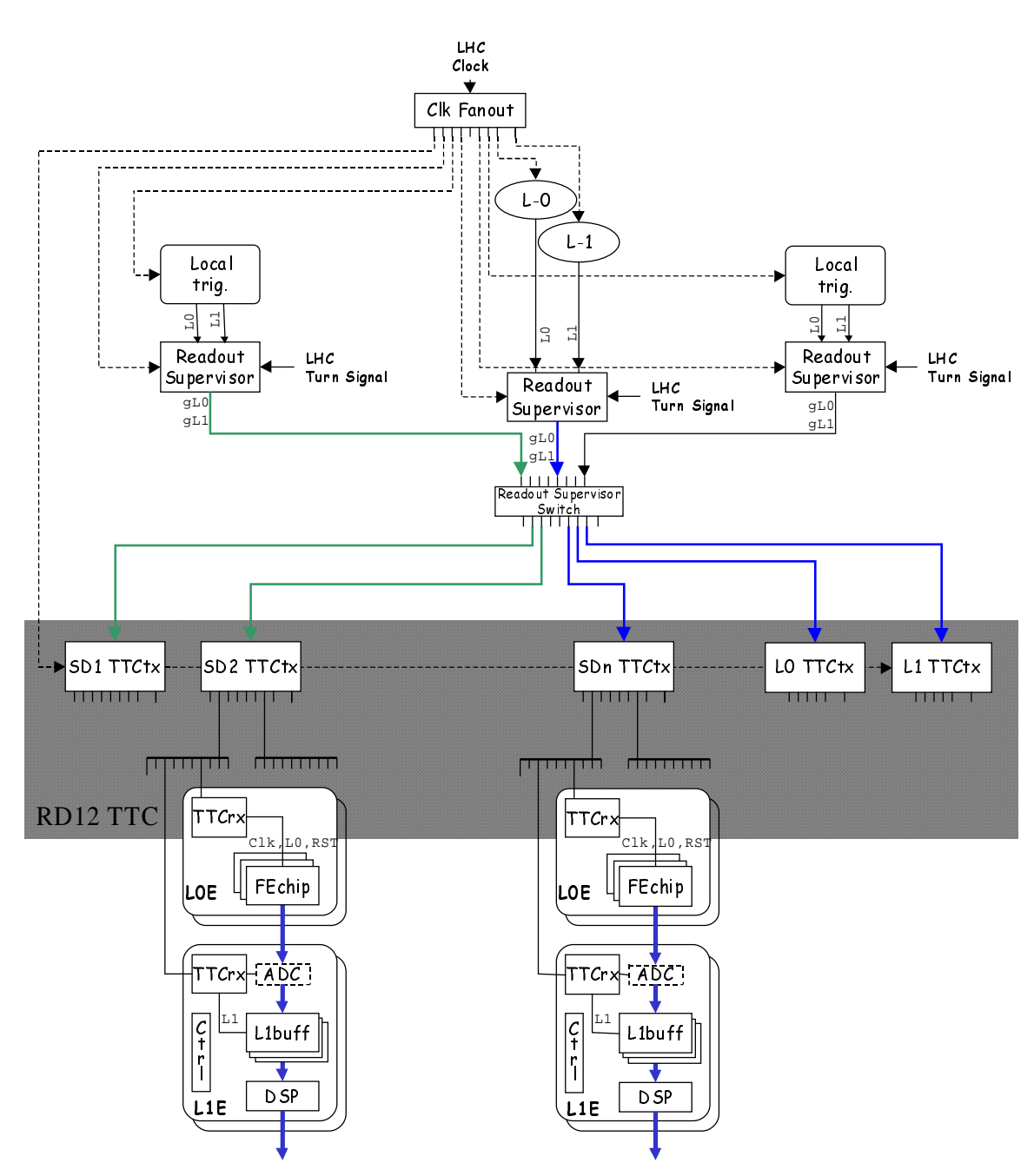




\section{IHChDAQ Functional Components}

$\square$ Readout Units (RUs)

$>$ Multiplex Front-end links onto Readout Network links

$>$ Merge input fragments to one output fragment

$\square$ Subfarm Controllers (SFCS)

$>$ assemble event fragments arriving from RUs to complete events and send them to one of the CPUs connected

$>$ Load balancing among the CPUs connected

$\square$ Readout Network

$>$ provide connectivity between RUs and SFCs for event-building

$>$ provide necessary bandwidth (4 GB/sec sustained)

$\square$ CPU farm

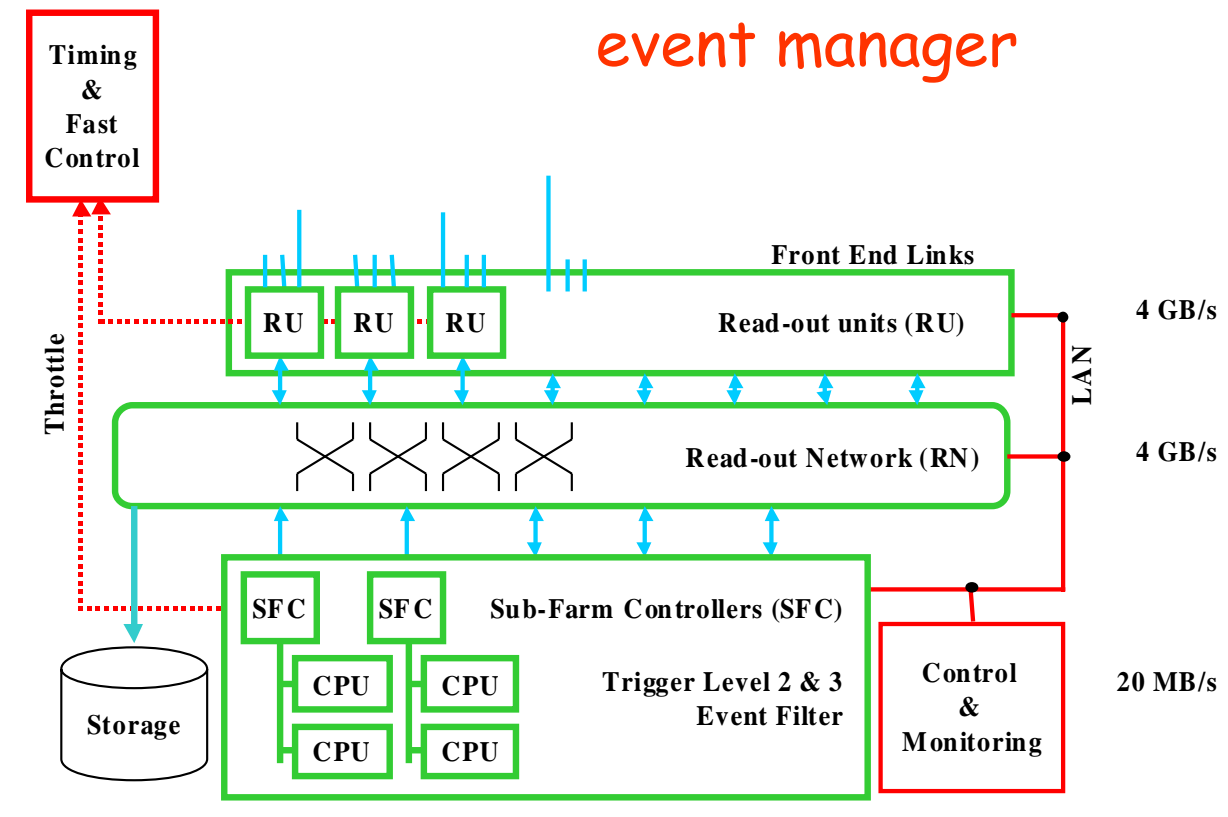

$>$ execute the high level trigger algorithms

- Level-2 (Input rate: $40 \mathrm{kHz}$, Output rate: $5 \mathrm{kHz}$ )

- Level-3 (Input rate: $5 \mathrm{kHz}$, Output rate: $\sim 100 \mathrm{~Hz}$ )

$>\sim 2000$ processors (à 1000 MIPS) 


\section{LHCb Control System}

Common integrated controls system

$>$ Detector controls (classical 'slow control')

$\rightarrow$ High voltage

$\rightarrow$ Low voltage

$\rightarrow$ Crates

$\rightarrow$ Temperatures

$\rightarrow$ Gas

$\rightarrow$ Alarm generation and handling

$\rightarrow$ etc.

$>D A Q$ controls

$\rightarrow$ Classical RUN control

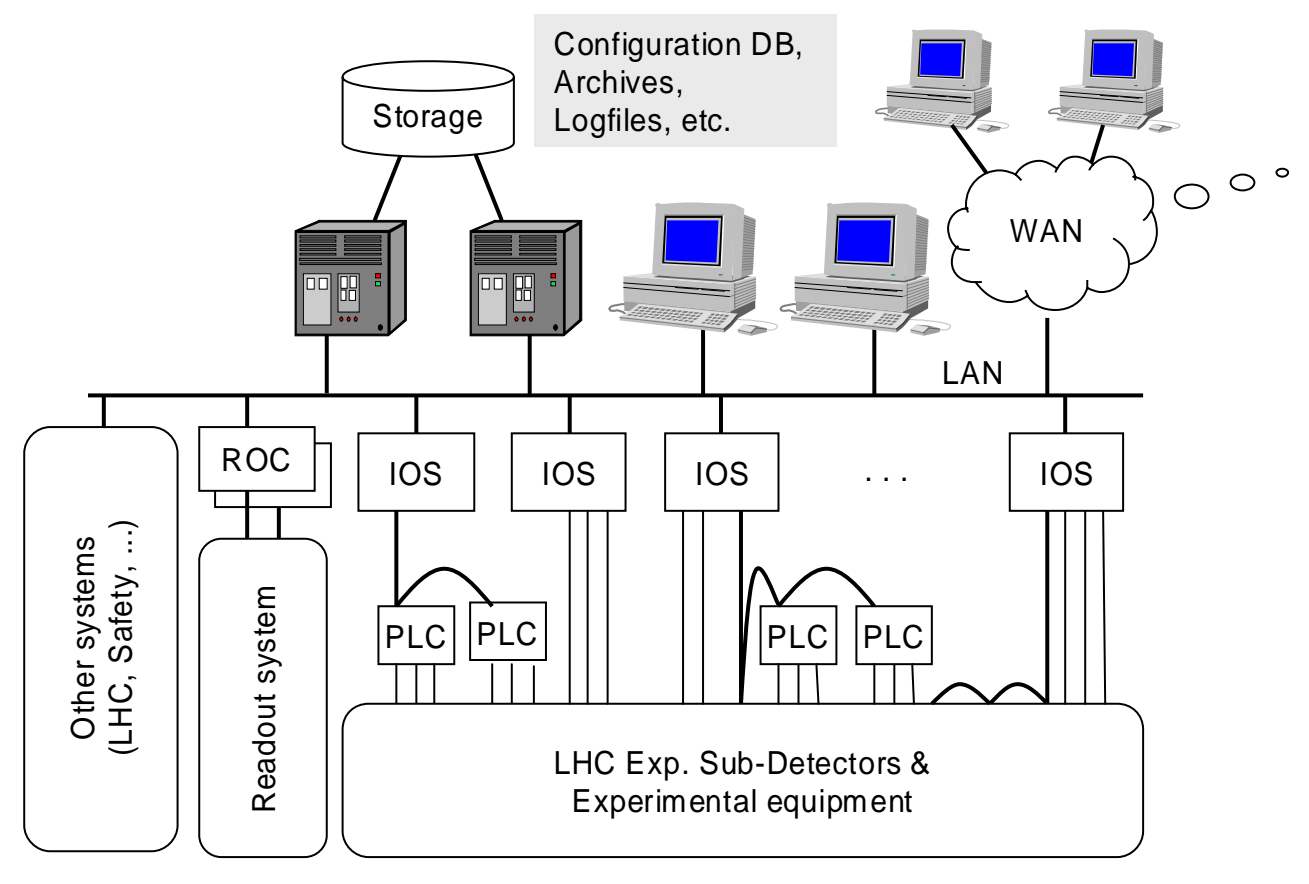

$\rightarrow$ Setup and configuration of all components (FE, Trigger,DAQ)

$\Rightarrow$ Monitoring

> Same system for both functions 


\section{KHCh Event-Building Network}

$\square$ Requirements

$>4 \mathrm{~GB} / \mathrm{s}$ sustained bandwidth

$>$ scalable

> expandable

$>\sim 100$ inputs (RUs)

$>\sim 100$ outputs (SFCs)

$>$ affordable and if possible commercial (COTS, Commodity?)

- Readout Protocol

Pure push-through protocol of complete events to one CPU of the farm

$\rtimes$ Simple hardware and software

$\rightarrow$ No central control $\rightarrow$ perfect scalability

ד Full flexibility for high-level trigger algorithms

Larger bandwidth needed

$\checkmark$ Avoiding buffer overflows via 'throttle' to TFC sub-system

\Static load balancing between RUs and SFCS 


\section{KHCb Event-Building Network Simulation}

a Simulated technology: Myrinet

- Nominal $1.28 \mathrm{~Gb} / \mathrm{s}$ (next year $2 \mathrm{~Gb} / \mathrm{s}$ )

$>$ Xon/Xoff flow control

$>$ Switches:

$\rightarrow$ ideal cross-bar

$\Rightarrow 8 \times 8$ maximum size (currently)

$\rightarrow$ wormhole routing

$\Rightarrow$ source routing

$\rightarrow$ No buffering inside switches

- Software used: "Ptolemy" discrete event framework

- Realistic traffic patterns

$>$ variable event sizes

$>$ event building traffic

- ATM studied in framework of RD31

- Work started on Gb Ethernet simulation

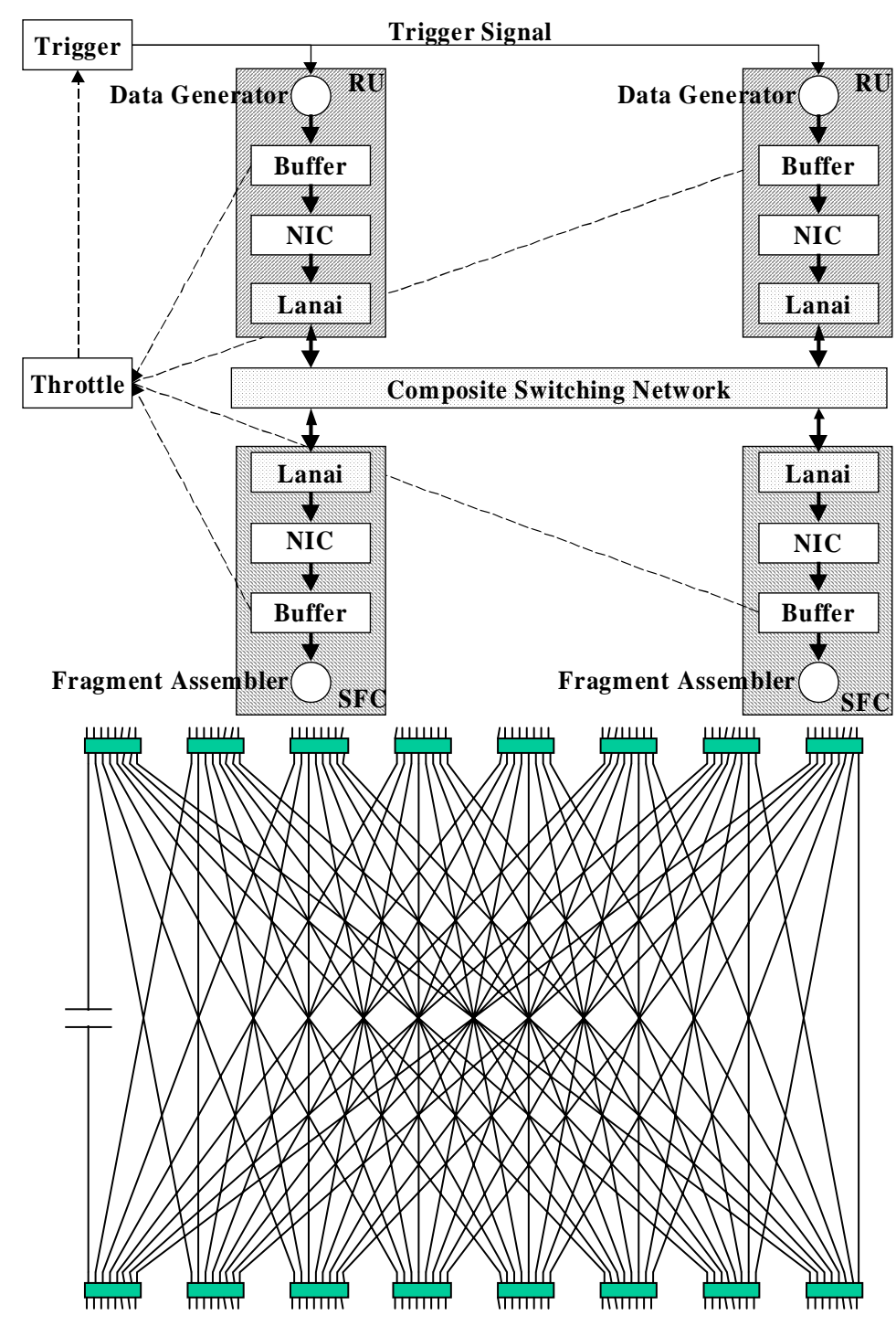

14 


\section{KHCh Network Simulation Results}

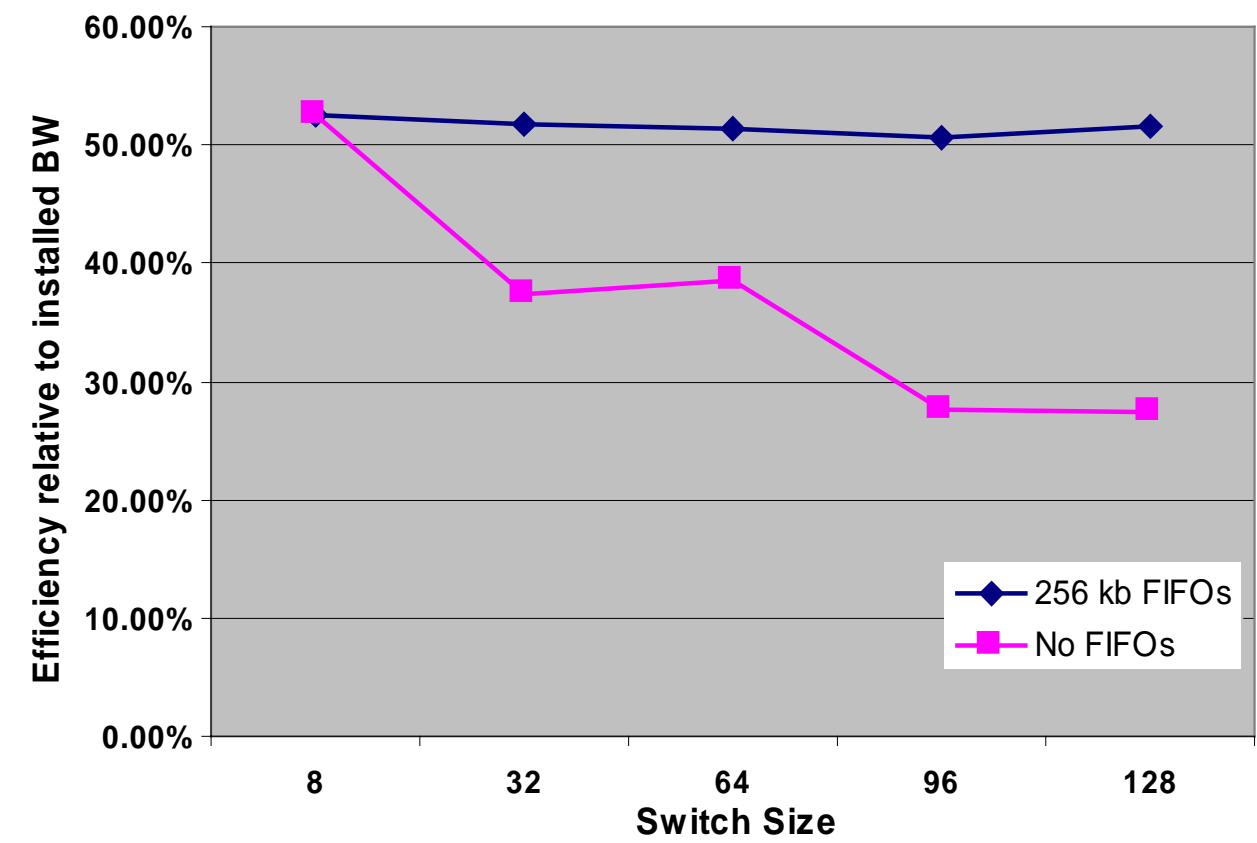

\begin{tabular}{|c|c|c|c|}
\hline Switch Size & Fifo Size & $\begin{array}{c}\text { Switching } \\
\text { Levels }\end{array}$ & Efficiency \\
\hline $8 \times 8$ & N A & 1 & $52.5 \%$ \\
\hline $32 \times 32$ & 0 & 2 & $37.3 \%$ \\
\hline $32 \times 32$ & $256 \mathrm{kB}$ & 2 & $51.8 \%$ \\
\hline $64 \times 64$ & 0 & 2 & $38.5 \%$ \\
\hline $64 \times 64$ & $256 \mathrm{kB}$ & 2 & $51.4 \%$ \\
\hline $96 \times 96$ & 0 & 3 & $27.6 \%$ \\
\hline $96 \times 96$ & $256 \mathrm{kB}$ & 3 & $50.7 \%$ \\
\hline $128 \times 128$ & 0 & 3 & $27.5 \%$ \\
\hline $128 \times 128$ & $256 \mathrm{kB}$ & 3 & $51.5 \%$ \\
\hline
\end{tabular}

-FIFO buffers between switching levels allow to recover scalability

-50 \% efficiency "Law of nature"

-Results don't depend strongly on specific technology (Myrinet), but rather on characteristics (flow control, Buffering) 


\section{KHCb Summary}

- LHCb is a special purpose experiment to study $C P$ violation

$\square$ FE Electronics split in two to cope with two levels of hardware triggers

$\square D A Q$ is designed with simplicity and maintainability in mind

$>$ Push readout protocol $\rightarrow$ Simple, e.g. No central event manager

$>$ Harder bandwidth requirements on readout network

$>$ Simulations suggest that readout network can be realized by adding FIFO buffers between levels of switching elements

$\square$ Unified approach to Controls

- Same basic infrastructure for detector controls and DAQ controls

$>$ Both aspects completely integrated but operationally independent 


\section{KHChProject Organization}

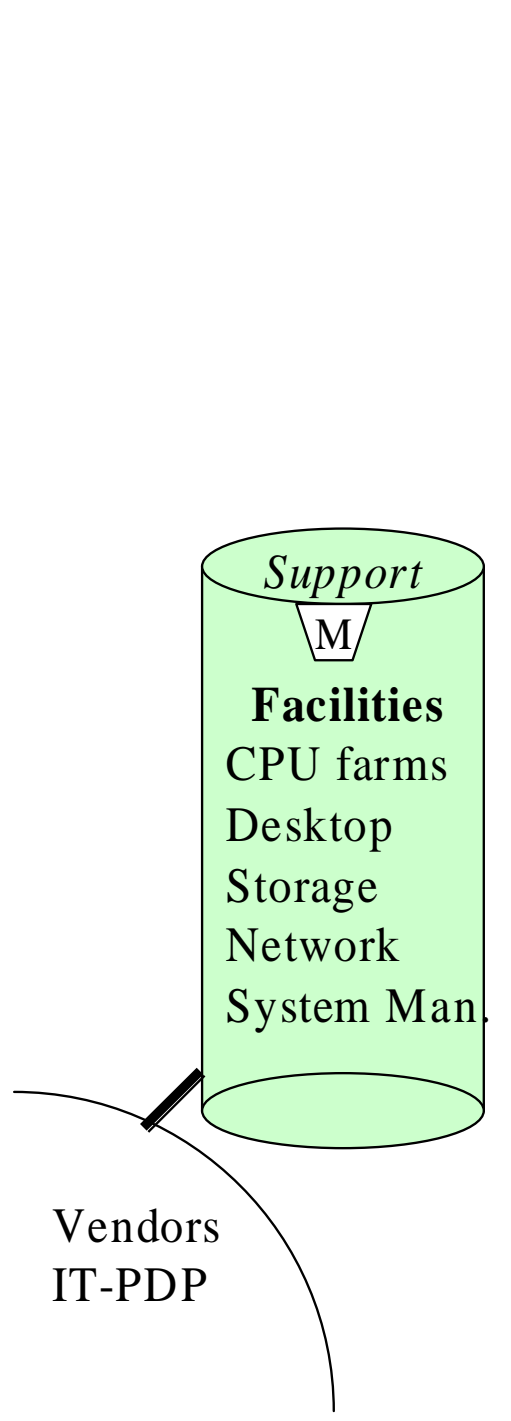

INSTR 99, Hamamatsu, 15-18 November 1999

\section{Steering Group \\ $\mathrm{M}, \mathrm{M}$ C ..}

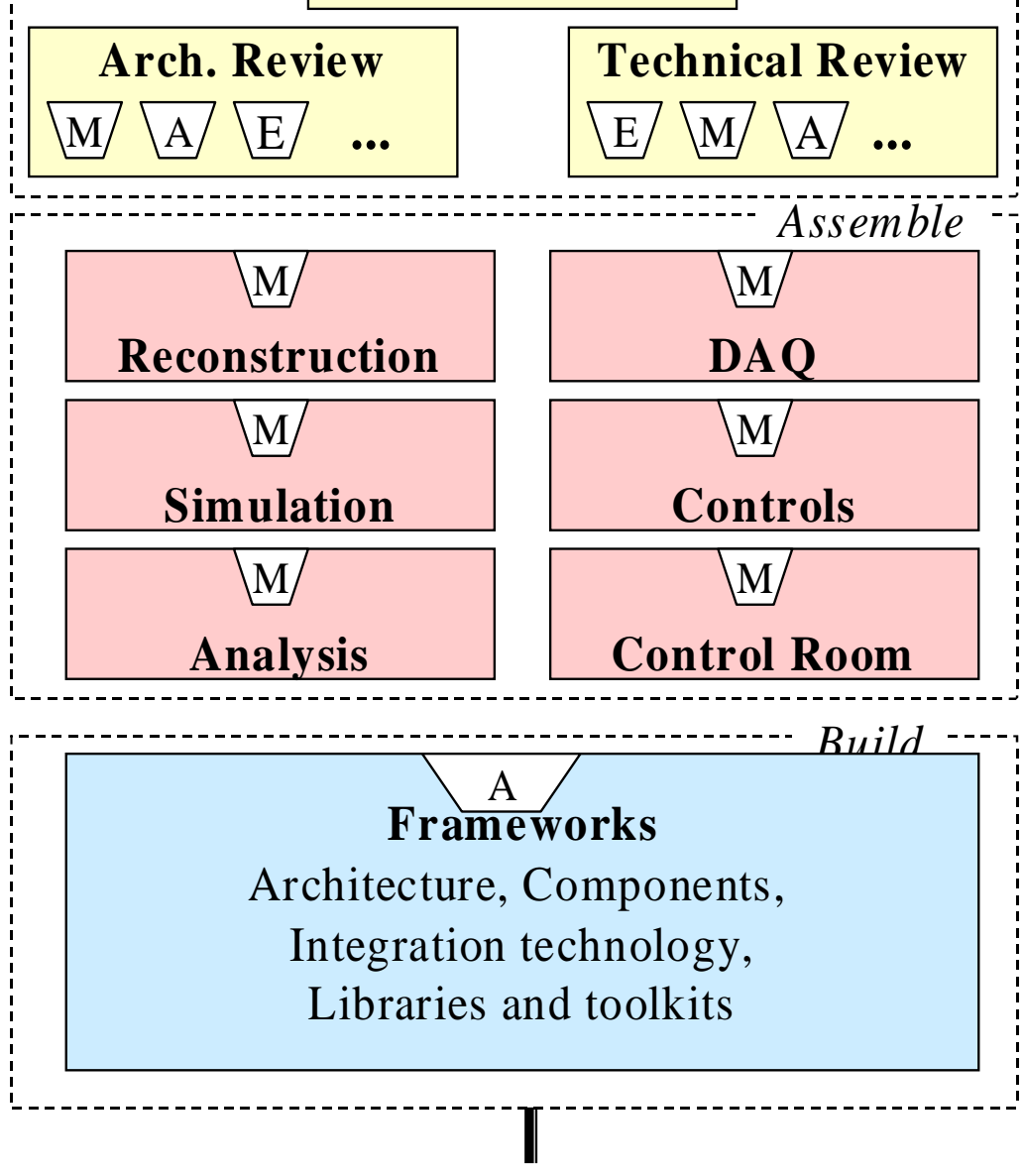

Vendors, IT-ASD
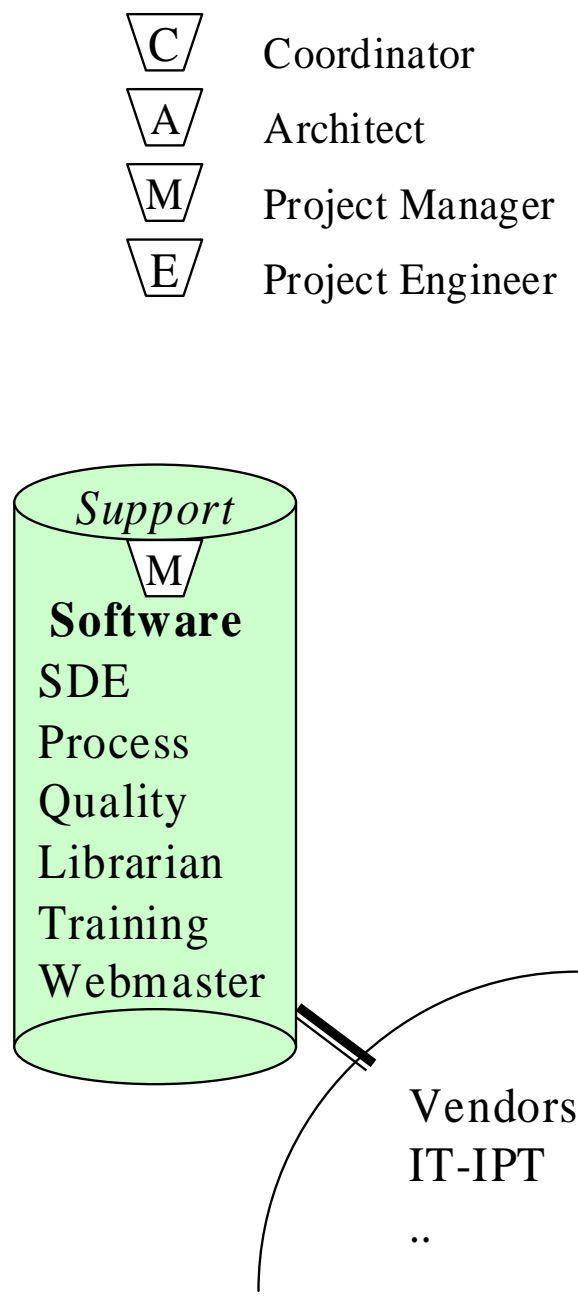